\title{
An Analytic Network Process based Decision Making Framework for Manager Selection
}

\author{
[Nasrullah khan,Ikhtiar khan, Ali Raza]
}

\begin{abstract}
The present and future of an organization can be defined in term of viability, sustainability and continuous progress. These terms depends on manager's role. The criticality of manager's role can further increase by the tough competition that most organizations have in today's business. To handle the complexity of manager selection process, this study proposes an MCDM decision making framework to model relative preferences of different manager characteristics by applying network approach. The framework consists of different layers where criteria and alternates can be specified. The network approach increases the relative preferences among different criteria.

Keywords- MCDM, manager characteristics, relative preferences.
\end{abstract}

\section{Introduction}

The Human Resource Management (HRM) is the process of organizing, managing and leading organizations in optimum way. In this extreme competitive business environment where most of organizations are applying very innovative approaches for achieving their objectives, the tracing of opportunity needs very technical and innovative mechanism. To enhance competitive advantages in this new knowledge economy era, organizations should be high competitive in human resources [1].The personal characteristics like skills, capabilities, knowledge and others are very critical to organization growth. Personnel selection is the process of choosing individuals who match the qualifications required to perform a defined job in the best way [2]. The human resource management process can be optimized by mature personal selection process. The process of personal selection determine the personal input quality play a key roles in human resource management [3]. When the personal selection process is precise and it matches the company circumstances then mangers can very easily optimize production costs and organization corporative goals are easily achieved [4].

\footnotetext{
Nasrullah khan

Northern university, Nowshera, KPK

Pakistan
}

Ikhtiar khan

Islamic international university, Islamabad

Ali, Raza

NUST Pakistan
The main objective of the selection process is to analyze differences among different candidates and to predict future performances [5]. The personal selection process becomes more critical when the candidates are managers. In organizations, there are different levels of management i.e tactical, operational and strategic. During each level managers have to design and monitor the organization policies and business processes. The decision making depends on manager knowledge and capabilities and affect the organization growth. The managers selection process is multi criterion where subjectivity, impreciseness exist. To model such scenario, this paper proposed an MCDM approach using Analytic Network Process which is one of the MCDM approaches. The ANP enhance the precision of relative preferences by applying network approach. The rest of the paper is organized as follows: section 2 explains related work, 3 shows proposed framework, 4 gave research method and section 5, 6 describe results and future work respectively.

\section{Related Work}

Many decision-making frameworks have been proposed by researchers for human resource selection. Many techniques from operation research and AI (artificial intelligence) were used to facilitate this decision problem. Hen and Cheng [6] proposed a fuzzy MCDM method in order to select project manager in information system domain. $\mathrm{Bi}$ and Zhang [7] did an analysis of project manager illegibility. They applied the Fuzzy AHP method based on triangular fuzzy numbers to prioritize different project managers as candidates. The fuzzy AHP method model the hierarchical structure of the problem with wit narrowing the membership function. Zhao et al. [9] did an extensive assessment by analyzing the project manager selection problem. Zavadskas et al. [10] developed multi criteria methodology for project manager selection based on grey criteria. Rashidi et al. [11] combined fuzzy systems, ANNs, and genetic algorithm for choosing a qualified project manager.

\section{Decision Making Framework}

The selection of project manager is very critical decision because the business sustainability depends 
on project manager role. This problem is multi criterion because many characteristics have to analyze. This framework consists of different layers for criteria, sub-criteria and alternates.

\subsection{Selection Criteria}

A through literature survey was executed in order to find project manager characteristics and to fulfill the requirements of decision making. These factors have not static nature but depend on the domain for which the project manager is selected. For example, different criteria should be considered for salesmen, IT systems developers or factory workers [12]. The following are managerial skills to be considered [12]: (1) creativity/innovation, (2) problem solving/decision making,(3)conflict management/negotiation,(4)empowerment/delegation ,(5) strategic planning, (6) specific presentation skills, (7) communication skill, (8) team management, (9) diversity management, (10) self management, (11) professional experience and (12) educational background.

\subsection{Analytic Network Process}

The MCDM approaches are excellent tools in handling decision making in situation subjectivity, human judgment exist. In business organization, the decision making is very complex due to conflicting interests and tough competitive environment. This method used network approach to model subjectivity, human judgment more precisely. This technique uses inter dependency among all nodes of network. The Analytic Network Process (ANP) is updated version of Analytic Hierarchy Process (AHP) [13]. In case of AHP, we use hierarchical structure to model the problem at hand but ANP provide an influence network of clusters and nodes contained within the clusters [13]. Figure 1 shows the ANP network structure

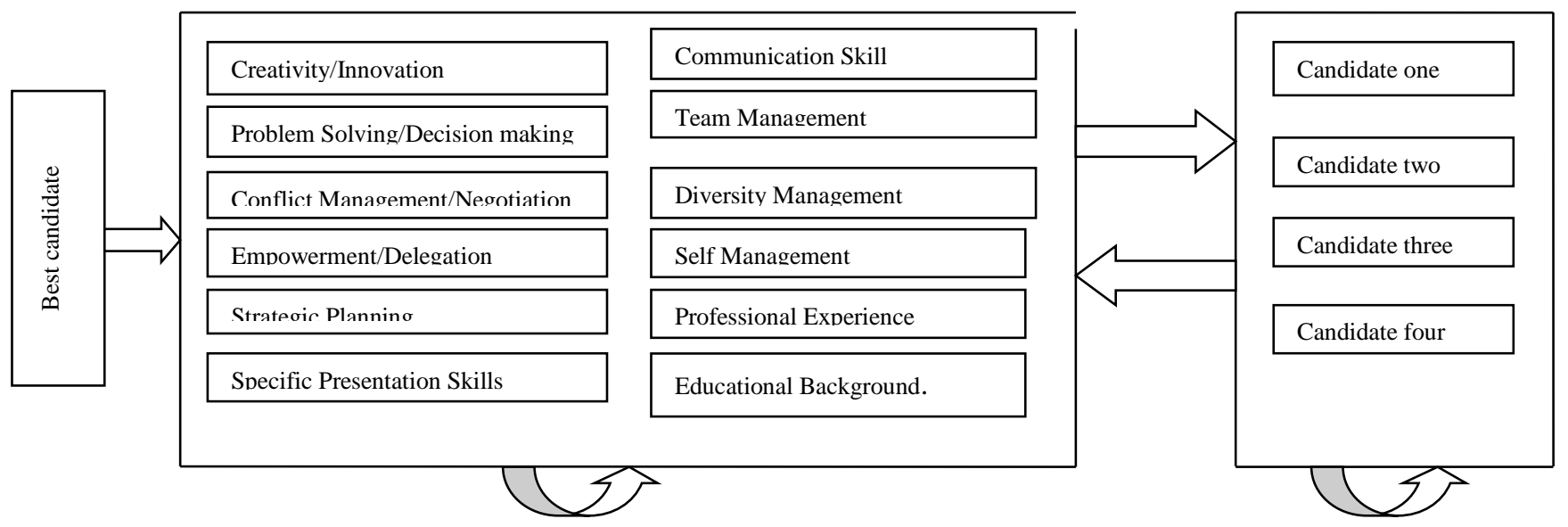

Fig 1. ANP Network Process

\section{Research Methodology}

Interpretivism provide input to this study i.e. realties can be constructed from social processes, and same reality have multiple interpretations and scientific research is time and context dependent [14].

\subsection{Sample Selection}

Decision-making is complex phenomenon in organization and these complexity exponentially increases when there is tough competition.

\subsection{Questionnaire Design}

The most challenging task in management research is to design questionnaire which collect more relevant and precise data. The questionnaires have to be designed very short in order to increase response rate. In this study AHP format was used for designing questions in questionnaire [15]. To keep the length of questionnaire short, transitive and reciprocal mathematical properties were used. Figure 2 shows format of the question used for AHP. Table 1 shows satty's scale which measure relative importance of one factor over other.

$\begin{array}{lllllllll}9 & 7 & 5 & 3 & \text { equal } & 3 & 5 & 7 & 9\end{array}$

Extreme preference

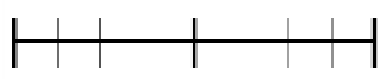

Extreme preference

Fig 2. AHP questionnaire format

\subsection{Research Questions}

In this study following research questions were investigated:

Q1. What is relative importance of criteria used for manager's selection in organizations? 
Proc. of the Third Intl. Conf. Advances in Social Science, Economics and Management Study- SEM 2015 Copyright $(\odot$ Institute of Research Engineers and Doctors, USA .All rights reserved.

ISBN:978-1-63248-063-7 doi: 10.15224/978-1-63248-063-7-44

Q2. Which candidate is more illegible for this position?

\section{Results}

The objective of this research work to analyze quantitatively the managers' selection problem. This study finds the relative importance of different criteria used for managers' selection in different organizations and on the basis of this evaluation the candidates for manager position have been prioritized. Table I, II and III shows the Supermatrix, weighted super matrix and limit matrix respectively.

Table I super matrix

\begin{tabular}{|c|c|c|c|c|c|c|c|c|c|c|c|c|c|c|c|c|}
\hline & 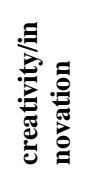 & 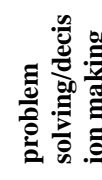 & 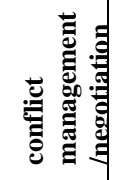 & 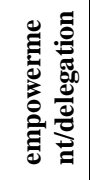 & 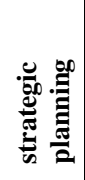 & 弟产 & 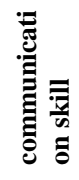 & 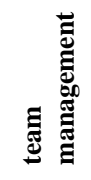 & 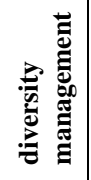 & 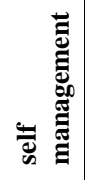 & 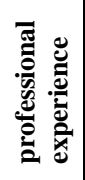 & 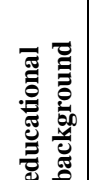 & 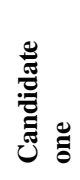 & 节 & 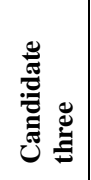 & 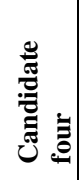 \\
\hline creativity/innovation & 0 & 0.09 & 0.10 & 0.07 & 0.09 & 0.07 & 0.08 & 0.09 & 0.07 & 0.08 & 0.07 & 0.77 & 0.08 & 0.08 & 0.74 & $\begin{array}{l}0.0 \\
7\end{array}$ \\
\hline problem solving/decision & .19 & 0 & 0.23 & 0.18 & 0.20 & 0.16 & 0.18 & 0.19 & 0.18 & 0.18 & 0.18 & 0.18 & 0.17 & 0.17 & 0.17 & $\begin{array}{l}0.1 \\
7\end{array}$ \\
\hline $\begin{array}{l}\text { conflict } \\
\text { management/negotiation }\end{array}$ & 0.23 & 0.25 & 0 & 0.22 & 0.25 & 0.22 & 0.23 & 0.24 & 0.22 & 0.23 & 0.22 & 0.22 & 0.17 & 0.17 & 0.17 & $\begin{array}{l}0.1 \\
7\end{array}$ \\
\hline empowerment/delegation & 0.02 & 0.02 & 0.02 & 0 & 0.02 & 0.02 & 0.02 & 0.02 & 0.02 & 0.02 & 0.02 & 0.02 & 0.02 & 0.02 & 0.02 & $\begin{array}{l}0.0 \\
2 \\
\end{array}$ \\
\hline strategic planning & 0.16 & 0.19 & 0.19 & 0.15 & 0 & 0.22 & 0.15 & 0.17 & 0.14 & 0.16 & 0.15 & 0.15 & 0.15 & 0.15 & 0.15 & $\begin{array}{l}0.1 \\
5 \\
\end{array}$ \\
\hline $\begin{array}{l}\text { Specific presentation } \\
\text { skills }\end{array}$ & 0.08 & 0.09 & 0.09 & 0.07 & 0.09 & 0 & 0.07 & 0.08 & 0.07 & 0.07 & 0.07 & 0.07 & 0.07 & 0.07 & 0.07 & $\begin{array}{l}0.0 \\
7 \\
\end{array}$ \\
\hline communication skill & 0.06 & 0.07 & 0.07 & 0.05 & 0.07 & 0.06 & 0 & 0.54 & 0.55 & 0.54 & 0.55 & 0.54 & 0.54 & 0.54 & 0.54 & $\begin{array}{l}0.5 \\
4\end{array}$ \\
\hline team management & 0.13 & 0.15 & 0.15 & 0.12 & 0.13 & 0.13 & 0.13 & 0 & 0.13 & 0.12 & 0.12 & 0.12 & 0.12 & 0.12 & 0.12 & $\begin{array}{l}0.1 \\
2\end{array}$ \\
\hline diversity & 0.04 & 0.04 & 0.04 & 0.04 & 0.04 & 0.04 & 0.04 & 0.04 & 0 & 0.04 & 0.04 & 0.04 & 0.04 & 0.04 & 0.04 & $\begin{array}{l}0.0 \\
4\end{array}$ \\
\hline self management & 0.04 & 0.04 & 0.04 & 0.04 & 0.04 & 0.04 & 0.04 & 0.04 & 0.04 & 0 & 0.04 & 0.04 & 0.04 & 0.04 & 0.04 & $\begin{array}{l}0.0 \\
4\end{array}$ \\
\hline professional experience & 0.03 & 0.03 & 0.03 & 0.03 & 0.03 & 0.03 & 0.03 & 0.03 & 0.03 & 0.03 & 0 & 0.03 & 0.03 & 0.03 & 0.03 & $\begin{array}{l}0.0 \\
3 \\
\end{array}$ \\
\hline educational background & 0.02 & 0.02 & 0.02 & 0.02 & 0.02 & 0.02 & 0.02 & 0.02 & 0.02 & 0.02 & 0.02 & 0 & 0.02 & 0.02 & 0.02 & $\begin{array}{l}0.0 \\
2 \\
\end{array}$ \\
\hline Candidate one & 0.57 & 0.57 & 0.57 & 0.57 & 0.57 & 0.57 & 0.57 & 0.57 & 0.57 & 0.57 & 0.57 & 0.57 & 0 & 0.57 & 0.57 & $\begin{array}{l}0.5 \\
7 \\
\end{array}$ \\
\hline Candidate two & 0.26 & 0.26 & 0.26 & 0.26 & 0.26 & 0.26 & 0.26 & 0.26 & 0.26 & 0.26 & 0.26 & 0.26 & 0.26 & 0 & 0.26 & $\begin{array}{l}0.2 \\
6\end{array}$ \\
\hline Candidate three & 0.12 & 0.12 & 0.12 & 0.12 & 0.12 & 0.12 & 0.12 & 0.12 & 0.12 & 0.12 & 0.12 & 0.12 & 0.12 & 0.12 & 0 & $\begin{array}{l}0.1 \\
2\end{array}$ \\
\hline Candidate four & 0.06 & 0.06 & 0.06 & 0.06 & 0.06 & 0.06 & 0.06 & 0.06 & 0.06 & 0.06 & 0.06 & 0.06 & 0.06 & 0.06 & 0.06 & 0 \\
\hline
\end{tabular}

Table II. Weighted Supermatrix

\begin{tabular}{|c|c|c|c|c|c|c|c|c|c|c|c|c|c|c|c|c|}
\hline & 总 & 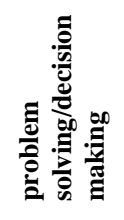 & 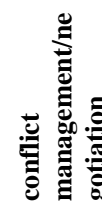 & 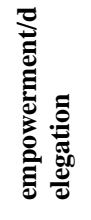 & 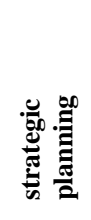 & 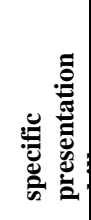 & |气 & 氧 & 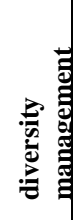 & 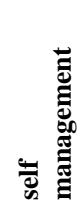 & 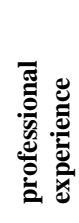 & 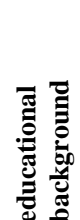 & 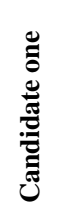 & 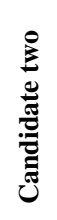 & 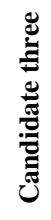 & 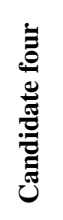 \\
\hline creativity/innovation & 0 & 0.04 & 0.04 & 0.04 & 0.04 & 0.04 & $\begin{array}{l}0.0 \\
4\end{array}$ & $\begin{array}{l}0.0 \\
4\end{array}$ & $\begin{array}{l}0.0 \\
4\end{array}$ & 0.04 & 0.04 & 0.04 & $\begin{array}{l}0.0 \\
4\end{array}$ & $\begin{array}{l}0.0 \\
4\end{array}$ & $\begin{array}{l}0 . \\
04\end{array}$ & 0.04 \\
\hline problem solving/decision & 0.02 & 0 & 0.092 & 0.092 & 0.092 & $\begin{array}{l}0.09 \\
2\end{array}$ & $\begin{array}{l}0.0 \\
92\end{array}$ & $\begin{array}{l}0.0 \\
92\end{array}$ & $\begin{array}{l}0.0 \\
92\end{array}$ & $\begin{array}{l}0.09 \\
2\end{array}$ & $\begin{array}{l}0.09 \\
2\end{array}$ & $\begin{array}{l}0.09 \\
2\end{array}$ & $\begin{array}{l}0.0 \\
92\end{array}$ & $\begin{array}{l}0.0 \\
92\end{array}$ & $\begin{array}{l}0 . \\
09 \\
2\end{array}$ & $\begin{array}{l}0.09 \\
2\end{array}$ \\
\hline conflict management/negotiation & 0.12 & 0.12 & 0 & 0.12 & 0.12 & 0.12 & $\begin{array}{l}0.1 \\
2 \\
\end{array}$ & $\begin{array}{l}0.1 \\
2 \\
\end{array}$ & $\begin{array}{l}0.1 \\
2 \\
\end{array}$ & 0.12 & 0.12 & 0.12 & $\begin{array}{l}0.1 \\
2 \\
\end{array}$ & $\begin{array}{l}0.1 \\
2 \\
\end{array}$ & $\begin{array}{l}0 . \\
12 \\
\end{array}$ & 0.12 \\
\hline empowerment/delegation & $\begin{array}{l}0.00 \\
8 \\
\end{array}$ & 0.008 & 0.008 & 0 & 0.008 & $\begin{array}{l}0.00 \\
8\end{array}$ & $\begin{array}{l}0.0 \\
08\end{array}$ & $\begin{array}{l}0.0 \\
08\end{array}$ & $\begin{array}{l}0.0 \\
08\end{array}$ & $\begin{array}{l}0.00 \\
8\end{array}$ & $\begin{array}{l}0.00 \\
8\end{array}$ & $\begin{array}{l}0.00 \\
8\end{array}$ & $\begin{array}{l}0.0 \\
08\end{array}$ & $\begin{array}{l}0.0 \\
08\end{array}$ & $\begin{array}{l}0 . \\
00 \\
8 \\
\end{array}$ & $\begin{array}{l}0.00 \\
8\end{array}$ \\
\hline strategic planning & 0.07 & 0.09 & 0.09 & 0.07 & 0 & 0.11 & $\begin{array}{l}0.0 \\
7\end{array}$ & $\begin{array}{l}0.0 \\
8\end{array}$ & $\begin{array}{l}0.0 \\
7\end{array}$ & 0.08 & 0.08 & 0.07 & $\begin{array}{l}0.0 \\
8\end{array}$ & $\begin{array}{l}0.0 \\
7\end{array}$ & $\begin{array}{l}0 . \\
07\end{array}$ & 0.07 \\
\hline
\end{tabular}


Proc. of the Third Intl. Conf. Advances in Social Science, Economics and Management Study- SEM 2015 Copyright (C) Institute of Research Engineers and Doctors, USA .All rights reserved.

ISBN:978-1-63248-063-7 doi: 10.15224/978-1-63248-063-7-44

\begin{tabular}{|c|c|c|c|c|c|c|c|c|c|c|c|c|c|c|c|c|}
\hline specific presentation skills & 0.04 & 0.04 & 0.04 & 0.04 & 0.04 & 0 & $\begin{array}{l}0.0 \\
4\end{array}$ & $\begin{array}{l}0.0 \\
4\end{array}$ & $\begin{array}{l}0.0 \\
4\end{array}$ & 0.04 & 0.04 & 0.04 & $\begin{array}{l}0.0 \\
4\end{array}$ & $\begin{array}{l}0.0 \\
4\end{array}$ & $\begin{array}{l}0 . \\
04\end{array}$ & 0.04 \\
\hline communication skill & 0.03 & 0.03 & 0.03 & 0.03 & 0.03 & 0.03 & 0 & $\begin{array}{l}0.0 \\
3 \\
\end{array}$ & $\begin{array}{l}0.0 \\
3 \\
\end{array}$ & 0.03 & 0.03 & 0.03 & $\begin{array}{l}0.0 \\
3 \\
\end{array}$ & $\begin{array}{l}0.0 \\
3 \\
\end{array}$ & $\begin{array}{l}0 . \\
03 \\
\end{array}$ & 0.03 \\
\hline team management & 0.08 & 0.08 & 0.08 & 0.08 & 0.08 & 0.08 & $\begin{array}{l}0.0 \\
8 \\
\end{array}$ & 0 & $\begin{array}{l}0.0 \\
8 \\
\end{array}$ & 0.08 & 0.08 & 0.08 & $\begin{array}{l}0.0 \\
8 \\
\end{array}$ & $\begin{array}{l}0.0 \\
8 \\
\end{array}$ & $\begin{array}{l}0 . \\
08 \\
\end{array}$ & 0.08 \\
\hline diversity management & 0.02 & 0.02 & 0.02 & 0.02 & 0.02 & 0.02 & $\begin{array}{l}0.0 \\
2 \\
\end{array}$ & $\begin{array}{l}0.0 \\
2 \\
\end{array}$ & 0 & 0.02 & 0.02 & 0.02 & $\begin{array}{l}0.0 \\
2 \\
\end{array}$ & $\begin{array}{l}0.0 \\
2 \\
\end{array}$ & $\begin{array}{l}0 . \\
02 \\
\end{array}$ & 0.02 \\
\hline self management & 0.02 & 0.02 & 0.02 & 0.02 & 0.02 & 0.02 & $\begin{array}{l}0.0 \\
2\end{array}$ & $\begin{array}{l}0.0 \\
2\end{array}$ & $\begin{array}{l}0.0 \\
2\end{array}$ & 0 & 0.02 & 0.02 & $\begin{array}{l}0.0 \\
2\end{array}$ & $\begin{array}{l}0.0 \\
2\end{array}$ & $\begin{array}{l}0 . \\
02\end{array}$ & 0.02 \\
\hline professional experience & 0.01 & 0.01 & 0.01 & 0.01 & 0.01 & 0.01 & $\begin{array}{l}0.0 \\
1\end{array}$ & $\begin{array}{l}0.0 \\
1\end{array}$ & $\begin{array}{l}0.0 \\
1\end{array}$ & 0.01 & 0 & 0.01 & $\begin{array}{l}0.0 \\
1\end{array}$ & $\begin{array}{l}0.0 \\
1\end{array}$ & $\begin{array}{l}0 . \\
01\end{array}$ & 0.01 \\
\hline educational background & 0.01 & 0.01 & 0.01 & 0.01 & 0.01 & 0.01 & $\begin{array}{l}0.0 \\
1\end{array}$ & $\begin{array}{l}0.0 \\
1\end{array}$ & $\begin{array}{l}0.0 \\
1\end{array}$ & 0.01 & 0.01 & 0 & $\begin{array}{l}0.0 \\
1\end{array}$ & $\begin{array}{l}0.0 \\
1\end{array}$ & $\begin{array}{l}0 . \\
01\end{array}$ & 0.01 \\
\hline Candidate one & 0.28 & 0.28 & 0.28 & 0.28 & 0.28 & 0.28 & $\begin{array}{l}0.2 \\
8\end{array}$ & $\begin{array}{l}0.2 \\
8\end{array}$ & $\begin{array}{l}0.2 \\
8\end{array}$ & 0.28 & 0.28 & 0.28 & 0 & $\begin{array}{l}0.2 \\
8\end{array}$ & $\begin{array}{l}0 . \\
28\end{array}$ & 0.28 \\
\hline Candidate two & 0.13 & 0.13 & 0.13 & 0.13 & 0.13 & 0.13 & $\begin{array}{l}0.1 \\
3 \\
\end{array}$ & $\begin{array}{l}0.1 \\
3 \\
\end{array}$ & $\begin{array}{l}0.1 \\
3 \\
\end{array}$ & 0.13 & 0.13 & 0.13 & $\begin{array}{l}0.1 \\
3 \\
\end{array}$ & 0 & $\begin{array}{l}0 . \\
13 \\
\end{array}$ & 0.13 \\
\hline Candidate three & 0.05 & 0.05 & 0.05 & 0.05 & 0.05 & 0.05 & $\begin{array}{l}0.0 \\
5 \\
\end{array}$ & $\begin{array}{l}0.0 \\
5 \\
\end{array}$ & $\begin{array}{l}0.0 \\
5 \\
\end{array}$ & 0.05 & 0.05 & 0.05 & $\begin{array}{l}0.0 \\
5 \\
\end{array}$ & $\begin{array}{l}0.0 \\
5 \\
\end{array}$ & 0 & 0.05 \\
\hline Candidate four & 0.02 & 0.02 & 0.02 & 0.02 & 0.02 & 0.02 & $\begin{array}{l}0.0 \\
2 \\
\end{array}$ & $\begin{array}{l}0.0 \\
2 \\
\end{array}$ & $\begin{array}{l}0.0 \\
2 \\
\end{array}$ & 0.02 & 0.02 & 0.02 & $\begin{array}{l}0.0 \\
2 \\
\end{array}$ & $\begin{array}{ll}0.0 \\
2 \\
\end{array}$ & $\begin{array}{l}0 . \\
02 \\
\end{array}$ & 0 \\
\hline
\end{tabular}

Table III. Limit matrix

\begin{tabular}{|c|c|c|c|c|c|c|c|c|c|c|c|c|c|c|c|c|}
\hline & 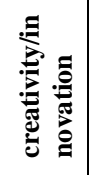 & 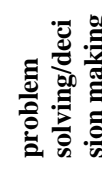 & 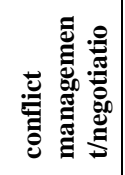 & 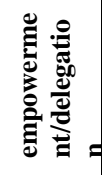 & 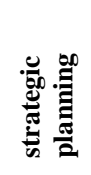 & 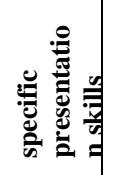 & 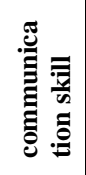 & 离 & 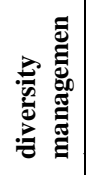 & 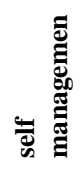 & 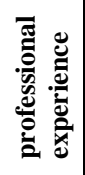 & 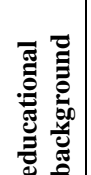 & 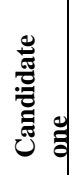 & 莺 & 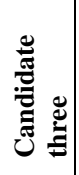 & 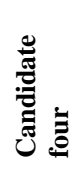 \\
\hline creativity/innovation & 0.04 & 0.04 & 0.04 & 0.04 & 0.04 & 0.04 & 0.04 & 0.04 & 0.04 & 0.04 & 0.04 & 0.04 & $\begin{array}{l}0.0 \\
4\end{array}$ & $\begin{array}{l}0.0 \\
4\end{array}$ & $\begin{array}{l}0.0 \\
4\end{array}$ & 0.04 \\
\hline problem solving/decision & $\begin{array}{l}0.08 \\
4\end{array}$ & 0.084 & 0.084 & 0.084 & 0.084 & 0.084 & $\begin{array}{l}0.08 \\
4\end{array}$ & 0.084 & $\begin{array}{l}0.08 \\
4\end{array}$ & $\begin{array}{l}0.08 \\
4\end{array}$ & $\begin{array}{l}0.08 \\
4\end{array}$ & $\begin{array}{l}0.08 \\
4\end{array}$ & $\begin{array}{l}0.0 \\
84\end{array}$ & $\begin{array}{l}0.0 \\
84\end{array}$ & $\begin{array}{l}0.0 \\
84\end{array}$ & $\begin{array}{l}0.08 \\
4\end{array}$ \\
\hline $\begin{array}{l}\text { conflict } \\
\text { management/negotiation }\end{array}$ & $\begin{array}{l}0.10 \\
1\end{array}$ & 0.101 & 0.101 & 0.101 & 0.101 & 0.101 & $\begin{array}{l}0.10 \\
1\end{array}$ & 0.101 & $\begin{array}{l}0.10 \\
1\end{array}$ & $\begin{array}{l}0.10 \\
1\end{array}$ & $\begin{array}{l}0.10 \\
1\end{array}$ & $\begin{array}{l}0.10 \\
1\end{array}$ & $\begin{array}{l}0.1 \\
01\end{array}$ & $\begin{array}{l}0.1 \\
01\end{array}$ & $\begin{array}{l}0.1 \\
01\end{array}$ & $\begin{array}{l}0.10 \\
1\end{array}$ \\
\hline empowerment/delegation & $\begin{array}{l}0.00 \\
8\end{array}$ & 0.008 & 0.008 & 0.008 & 0.008 & 0.008 & $\begin{array}{l}0.00 \\
8\end{array}$ & 0.008 & $\begin{array}{l}0.00 \\
8\end{array}$ & $\begin{array}{l}0.00 \\
8\end{array}$ & $\begin{array}{l}0.00 \\
8\end{array}$ & $\begin{array}{l}0.00 \\
8\end{array}$ & $\begin{array}{l}0.0 \\
08\end{array}$ & $\begin{array}{l}0.0 \\
08\end{array}$ & $\begin{array}{l}0.0 \\
08\end{array}$ & $\begin{array}{l}0.00 \\
8\end{array}$ \\
\hline strategic planning & $\begin{array}{l}0.07 \\
4\end{array}$ & 0.074 & 0.074 & 0.074 & 0.074 & 0.074 & $\begin{array}{l}0.07 \\
4\end{array}$ & 0.074 & $\begin{array}{l}0.07 \\
4\end{array}$ & $\begin{array}{l}0.07 \\
4\end{array}$ & $\begin{array}{l}0.07 \\
4\end{array}$ & $\begin{array}{l}0.07 \\
4\end{array}$ & $\begin{array}{l}0.0 \\
74\end{array}$ & $\begin{array}{l}0.0 \\
74\end{array}$ & $\begin{array}{l}0.0 \\
74\end{array}$ & $\begin{array}{l}0.07 \\
4\end{array}$ \\
\hline specific presentation skills & $\begin{array}{l}0.03 \\
8\end{array}$ & 0.038 & 0.038 & 0.038 & 0.038 & 0.038 & $\begin{array}{l}0.03 \\
8\end{array}$ & 0.038 & $\begin{array}{l}0.03 \\
8\end{array}$ & $\begin{array}{l}0.03 \\
8\end{array}$ & $\begin{array}{l}0.03 \\
8\end{array}$ & $\begin{array}{l}0.03 \\
8\end{array}$ & $\begin{array}{l}0.0 \\
38\end{array}$ & $\begin{array}{l}0.0 \\
38\end{array}$ & $\begin{array}{l}0.0 \\
38\end{array}$ & $\begin{array}{l}0.03 \\
8\end{array}$ \\
\hline communication skill & $\begin{array}{l}0.02 \\
9\end{array}$ & 0.029 & 0.029 & 0.029 & 0.029 & 0.029 & $\begin{array}{l}0.02 \\
9\end{array}$ & 0.029 & $\begin{array}{l}0.02 \\
9\end{array}$ & $\begin{array}{l}0.02 \\
9 \\
\end{array}$ & $\begin{array}{l}0.02 \\
9\end{array}$ & $\begin{array}{l}0.02 \\
9\end{array}$ & $\begin{array}{l}0.0 \\
29\end{array}$ & $\begin{array}{l}0.0 \\
29\end{array}$ & $\begin{array}{l}0.0 \\
29\end{array}$ & $\begin{array}{l}0.02 \\
9\end{array}$ \\
\hline team management & 0.07 & 0.061 & 0.061 & 0.061 & 0.061 & 0.061 & $\begin{array}{l}0.06 \\
1\end{array}$ & 0.061 & $\begin{array}{l}0.06 \\
1\end{array}$ & $\begin{array}{l}0.06 \\
1\end{array}$ & $\begin{array}{l}0.06 \\
1\end{array}$ & $\begin{array}{l}0.06 \\
1\end{array}$ & $\begin{array}{l}0.0 \\
61\end{array}$ & $\begin{array}{l}0.0 \\
61\end{array}$ & $\begin{array}{l}0.0 \\
61\end{array}$ & $\begin{array}{l}0.06 \\
1\end{array}$ \\
\hline diversity management & $\begin{array}{l}0.01 \\
9\end{array}$ & 0.019 & 0.019 & 0.019 & 0.019 & 0.019 & $\begin{array}{l}0.01 \\
9\end{array}$ & 0.019 & $\begin{array}{l}0.01 \\
9\end{array}$ & $\begin{array}{l}0.01 \\
9\end{array}$ & $\begin{array}{l}0.01 \\
9\end{array}$ & $\begin{array}{l}0.01 \\
9\end{array}$ & $\begin{array}{l}0.0 \\
19\end{array}$ & $\begin{array}{l}0.0 \\
19\end{array}$ & $\begin{array}{l}0.0 \\
19\end{array}$ & $\begin{array}{l}0.01 \\
9\end{array}$ \\
\hline self management & $\begin{array}{l}0.02 \\
2\end{array}$ & 0.022 & 0.022 & 0.022 & 0.022 & 0.022 & $\begin{array}{l}0.02 \\
2\end{array}$ & 0.022 & $\begin{array}{l}0.02 \\
2\end{array}$ & $\begin{array}{l}0.02 \\
2\end{array}$ & $\begin{array}{l}0.02 \\
2\end{array}$ & $\begin{array}{l}0.02 \\
2\end{array}$ & $\begin{array}{l}0.0 \\
22\end{array}$ & $\begin{array}{l}0.0 \\
22\end{array}$ & $\begin{array}{l}0.0 \\
22\end{array}$ & $\begin{array}{l}0.02 \\
2\end{array}$ \\
\hline professional experience & $\begin{array}{l}0.01 \\
4\end{array}$ & 0.014 & 0.014 & 0.014 & 0.014 & 0.014 & $\begin{array}{l}0.01 \\
4\end{array}$ & 0.014 & $\begin{array}{l}0.01 \\
4\end{array}$ & $\begin{array}{l}0.01 \\
4\end{array}$ & $\begin{array}{l}0.01 \\
4\end{array}$ & $\begin{array}{l}0.01 \\
4\end{array}$ & $\begin{array}{l}0.0 \\
14\end{array}$ & $\begin{array}{l}0.0 \\
14\end{array}$ & $\begin{array}{l}0.0 \\
14\end{array}$ & $\begin{array}{l}0.01 \\
4\end{array}$ \\
\hline educational background & 0.01 & 0.01 & 0.01 & 0.01 & 0.01 & 0.01 & 0.01 & 0.01 & 0.01 & 0.01 & 0.01 & 0.01 & $\begin{array}{l}0.0 \\
1\end{array}$ & $\begin{array}{l}0.0 \\
1\end{array}$ & $\begin{array}{l}0.0 \\
1\end{array}$ & 0.01 \\
\hline Candidate one & $\begin{array}{l}0.23 \\
3\end{array}$ & 0.233 & 0.233 & 0.233 & 0.233 & 0.233 & $\begin{array}{l}0.23 \\
3\end{array}$ & 0.233 & $\begin{array}{l}0.23 \\
3\end{array}$ & $\begin{array}{l}0.23 \\
3\end{array}$ & $\begin{array}{l}0.23 \\
3\end{array}$ & $\begin{array}{l}0.23 \\
3\end{array}$ & $\begin{array}{l}0.2 \\
33\end{array}$ & $\begin{array}{l}0.2 \\
33\end{array}$ & $\begin{array}{l}0.2 \\
33\end{array}$ & $\begin{array}{l}0.23 \\
3\end{array}$ \\
\hline Candidate two & $\begin{array}{l}0.16 \\
5\end{array}$ & 0.165 & 0.165 & 0.165 & 0.165 & 0.165 & $\begin{array}{l}0.16 \\
5\end{array}$ & 0.165 & $\begin{array}{l}0.16 \\
5\end{array}$ & $\begin{array}{l}0.16 \\
5\end{array}$ & $\begin{array}{l}0.16 \\
5\end{array}$ & $\begin{array}{l}0.16 \\
5\end{array}$ & $\begin{array}{l}0.1 \\
65\end{array}$ & $\begin{array}{l}0.1 \\
65\end{array}$ & $\begin{array}{l}0.1 \\
65\end{array}$ & $\begin{array}{l}0.16 \\
5\end{array}$ \\
\hline Candidate three & $\begin{array}{l}0.07 \\
1\end{array}$ & 0.071 & 0.071 & 0.071 & 0.071 & 0.071 & $\begin{array}{l}0.07 \\
1\end{array}$ & 0.071 & $\begin{array}{l}0.07 \\
1\end{array}$ & $\begin{array}{l}0.07 \\
1\end{array}$ & $\begin{array}{l}0.07 \\
1\end{array}$ & $\begin{array}{l}0.07 \\
1\end{array}$ & $\begin{array}{l}0.0 \\
71\end{array}$ & $\begin{array}{l}0.0 \\
71\end{array}$ & $\begin{array}{l}0.0 \\
71\end{array}$ & $\begin{array}{l}0.07 \\
1\end{array}$ \\
\hline Candidate four & $\begin{array}{l}0.03 \\
1\end{array}$ & 0.031 & 0.031 & 0.031 & 0.031 & 0.031 & $\begin{array}{l}0.03 \\
1\end{array}$ & 0.031 & $\begin{array}{l}0.03 \\
1\end{array}$ & $\begin{array}{l}0.03 \\
1\end{array}$ & $\begin{array}{l}0.03 \\
1\end{array}$ & $\begin{array}{l}0.03 \\
1\end{array}$ & $\begin{array}{l}0.0 \\
31\end{array}$ & $\begin{array}{l}0.0 \\
31\end{array}$ & $\begin{array}{l}0.0 \\
31\end{array}$ & $\begin{array}{l}0.03 \\
1\end{array}$ \\
\hline
\end{tabular}




\section{1prioritization of criteria}

The criteria used for the evaluation of candidates for manager position was prioritized on the basis of their relative importance. This prioritization is not static but depends on requirements of organization.

Table IV. Criteria prioritization

\begin{tabular}{|l|l|l|l|}
\hline S.no & Criteria & $\begin{array}{l}\text { Relative } \\
\text { importance }\end{array}$ & Ranking \\
\hline 1 & creativity/innovation & 0.04 & 5 \\
\hline 2 & problem solving/decision & 0.084 & 2 \\
\hline 3 & conflict management/negotiation & 0.101 & 1 \\
\hline 4 & empowerment/delegation & 0.008 & 12 \\
\hline 5 & strategic planning & 0.074 & 3 \\
\hline 6 & specific presentation skills & 0.038 & 6 \\
\hline 7 & communication skill & 0.029 & 7 \\
\hline 8 & team management & 0.061 & 4 \\
\hline 9 & diversity management & 0.019 & 9 \\
\hline 10 & self management & 0.022 & 8 \\
\hline 11 & professional experience & 0.014 & 10 \\
\hline 12 & educational background & 0.01 & 11 \\
\hline
\end{tabular}

\subsection{Candidate's prioritization}

The candidates were evaluated with respect to each other and with respect to relative importance of reported criteria. The final prioritized list of candidates which meets the reported criteria up to different extent is shown in table $\mathrm{V}$.

Table V. Candidate Prioritization

\begin{tabular}{|l|l|l|l|}
\hline S.no & Candidates & $\begin{array}{l}\text { Relative } \\
\text { value }\end{array}$ & Ranking \\
\hline 1 & Candidate one & 0.233 & 1 \\
\hline 2 & Candidate two & 0.165 & 2 \\
\hline 3 & Candidate three & 0.071 & 3 \\
\hline 4 & Candidate four & 0.031 & 4 \\
\hline
\end{tabular}

\section{Discussion, conclusion and future work}

The conflict management /negotiation and problem solving/decision making were most important and critical parameters to be consider during managers selection problem. The limitation of the study is, that different characteristics can be considered while selecting managers for different domains. The decision framework proposed in this study is domain independent. Candidates one has top position means that it satisfy the requirement up to maximum percentage. The other limitation of the study is that preferences were determined using classical set theory based on:"Yes" and "No". In future the precision of membership can be enhanced by fuzzy approaches and other approaches.

\section{References}

[1] H. T, Lin, " Personnel selection using analytic network process and fuzzy data envelopment analysis approaches", Computers \& Industrial Engineering, 59(4), 2010,pp.937-944

[2] M.Dursun, \& E. E. Karsak, "A fuzzy MCDM approach for personnel selection", Expert Systems with Applications, 37(6), 2010, pp.4324-4330.

[3] A.Balezzentis, ,T. Balezzentis, \& W. K.M.Brauers, ," Personnel selection based on computing with words and fuzzy MULTIMOORA", Expert Systems with Applications, 39(9), 2012, pp.7961-7967

[4] L.Can' os, \& V.Liern," Soft computing-based aggregation methods for human resource management", European Journal of Operational Research, 189(3),2008,pp. 669-681

[5] Z.G"ung"or, G.Serhadlıo glu, \& S. E. Kesen," A fuzzy AHP approach to personnel selection problem", Applied Soft Computing, 9(2),2009, pp.641-646.

[6] L.-S. Chen and C.-H. Cheng, "Selecting IS personnel use fuzzy GDSS based on metric distance method," European Journal of Operational Research, vol. 160, no. 3, pp. 803-820, 2005.

[7] X. Bi and A.-D. Zhang, "Application of fuzzy analytical hierarchy process in selecting a project manager," in Proceedings of the 13th International Conference on Management Science and Engineering (ICMSE '06), pp. 14171421, October 2006.

[8] Z. Hui,W. Xue-qing, Y. Gang, and Z. Li-fu, "Study on engineering project manager selection," in Proceedings of the 4th International Conference onWireless Communications, Networking and Mobile Computing (WiCOM '08), IEEE, 2008.

[9] L. Zhao, Y. Guo, and W. Cui, "The application of fuzzy comprehensive evaluation methods in the selection of a project manager," in Proceedings of the 4th International Conference on Computer Sciences and Convergence Information Technology (ICCIT '09), pp. 1387-1391, IEEE, November 2009.

[10] E.K. Zavadskas, Z. Turskis, J. Tamo saitiene, and V. Marina, "Multicriteria selection of project managers by applying grey criteria," Technological and Economic Development of Economy, vol. 14, no. 4, pp. 462-477, 2008.

[11] A. Rashidi, F. Jazebi, and I. Brilakis, "Neurofuzzy genetic system for selection of construction project managers," Journal of Construction Engineering and Management, vol. 137, no. 1, pp. 17-29, 2011.

[12] R. D. Arvey, \& J. E. Campion, "The employment Interview: A summary and review of recent research". Staff Psychology, vol $.35,1982$, pp.281-322.

[13] L.F WANG, The theory and algorithm of ANP, System Engineering Theories and Practices, pp.44-50, 2001.

[14] T.L. Saaty and T.L.VargasLG "Decision making with the analytic networking process", $2^{\text {nd }}$ ed, vol.195, Springer's International Series, New York,2013.

[15] A. Abid. "An Empirical Evaluation Of M-Payment Business Models Using Analytic Hierarchy Process And Sensitivity Analysis", International Symposium of the Analytic Hierarchy Process", Washington, D.C. June 29 - July 2, 2014 